Research Article

\title{
Event-Triggered Dissipative Filter Design for Semi-Markovian Jump Systems with Time-Varying Delays
}

\author{
Huiying Chen $(\mathbb{D}$, Zuxin Li $\mathbb{D}$, and Weifeng Xia \\ School of Engineering, Huzhou University, Huzhou, Zhejiang 313000, China \\ Correspondence should be addressed to Zuxin Li; lzx@zjhu.edu.cn
}

Received 26 August 2019; Revised 19 December 2019; Accepted 27 December 2019; Published 17 March 2020

Academic Editor: Libor Pekař

Copyright (C) 2020 Huiying Chen et al. This is an open access article distributed under the Creative Commons Attribution License, which permits unrestricted use, distribution, and reproduction in any medium, provided the original work is properly cited.

\begin{abstract}
This paper deals with the event-triggered dissipative filtering problem for semi-Markovian jump systems with time-varying delays. The purpose is to design a filter which guarantees that the filtering error system is not only stochastically stable but also satisfies dissipativity. First, based on Lyapunov-Krasovskii theory and matrix integral inequality, a sufficient condition is established for the existence of such a filter. Then, the codesign method of event-triggered matrices and the desired filter parameters is proposed. Finally, two numerical examples are given to illustrate the advantages and validity of the scheme developed in this paper.
\end{abstract}

\section{Introduction}

Over the past decades, Markovian jump systems (MJSs) have attracted significant attention by the research community mainly due to the fact that they have a powerful framework to model practical systems, such as economy [1], robotics [2], mechanics [3], and solar thermal receivers [4]. However, among the aforementioned works, the jump time of a Markov chain is a time-homogeneous exponential random variable. This result in the MJSs has many limitations in applications. To overcome this obstacle, a more wider class of systems with general sojourn time distributions, named semi-Markovian jump systems (SMJSs), have been studied in recent years. To mention a few, the problem of stability and stabilization for semi-Markovian jump systems was addressed in [5-7]. The controller design problem for semiMarkovian jump systems was investigated in [8-11]. In $[12,13]$, the filtering problem for semi-Markovian jump systems was considered.

It is known that time-delay phenomenon is commonly observed in real-world applications and usually deteriorates the stability of a control system [14-22]. As a consequence, increasing attention has been devoted to studying of SMJSs with time delays. For instance, the problem of finite-time $H_{\infty}$ synchronization for SMJSs with time-varying delays was reported in $[23,24]$. The resilient estimation problem for nonlinear descriptor SMJSs was probed in [25], where the T-S fuzzy model and time-varying delays are taken into account. In the case, when the actuator or sensor failures appear in the SMJSs, the reliable controller or filter was designed for delayed SMJSs in [26, 27], respectively. By applying a piecewise analysis method, a stochastic stability condition was established in [28], where the time-varying delays are dependent on system modes. However, the stability condition in [28] may be conservative because Jensen's inequality was used to bound the integral term, and thus there still exists some room to improve. This is the first motivation of this paper.

On the other hand, communication resource saving is also a significant issue for networked systems because of the bandwidth resource becomes more and more limited as the complexity of the network increase [29]. It has been shown that the event-triggered scheme is an effective way to solve this issue. Compared with a time-triggered scheme, not all of sampled data packets in the event-triggered scheme are transmitted through a communication network. Only when an event condition is satisfied, the sampled data packets are transmitted [30]. As a result, the unnecessary communication resources are saved in the event-triggered scheme. In recent years, the event-triggered scheme has been utilized in the controller and filter design problem for various dynamic systems [31-36]. As for SMJSs, the event-triggered leader- 
following consensus for a multiagent system with semiMarkovian jumping parameters was studied in [37]. In the case of discrete-time systems, an $H_{\infty}$ filter was designed in [38] for a class of semi-Markovian jumping neural networks, where the quantized measurements was used in order to save the network resource, although the dissipative filtering problem for SMJSs was discussed in [39], where timevarying delays were not be considered. Therefore, the eventtriggered filtering problem for SMJSs with time-varying delays should be further investigated. This is also a motivation to this paper.

Based on the above mentioned discussions, this paper considers the dissipative filter design problem for SMJSs with time-varying delays by using the event-triggered scheme. The main contributions of this paper can be highlighted as follows: (1) the dissipative filter design problem for SMJSs in the presence of time-varying delays is investigated by applying the event-triggered scheme. (2) Based on Lyapunov-Krasovskii theory and matrix integral inequality, a new stochastic stability criterion for SMJSs is established. (3) The method developed in this paper will be helpful for some further development involving this topic, such as nonfragile control and state estimation.

The rest part of the paper is organized as follows. In Section 2, we describe the problem formulation. Section 3 presents the event-triggered filter design scheme for the underlying system. Numerical examples and simulation results which demonstrate the effectiveness and advantages of the proposed scheme are provided in Section 4. The conclusion of this paper is made in Section 5.

Notations. Throughout this paper, a real symmetric matrix $X>0(X \geq 0)$ means that $X$ is positive definite (respectively, positive semidefinite) matrix; $X^{T}$ represents transpose of the matrix $X ; \mathbb{R}^{n}$ and $\mathbb{R}^{n \times m}$ stand for $n$-dimensional Euclidean space and set of all $n \times m$ real matrices; $I_{n}$ denotes the $n$-dimensional identity matrix; $0_{n}$ and $0_{n \times m}$ denote the $n$-dimensional zero matrix and $n \times m$-dimensional zero matrix; $\operatorname{sym}\{X\}$ denotes $X+X^{T}$; $\operatorname{diag}\{\cdots\}$ stands for a blockdiagonal matrix. The notation $*$ is used as an ellipsis for terms that are induced by symmetry; $L_{2}[0, \infty)$ is the space of the square-integrable vector function over $[0, \infty) ;|\cdot|$ denotes the Euclidean norm for vectors. Matrix dimensions, if not explicitly stated are assumed to be compatible for algebraic operations.

\section{Problem Statement and Preliminaries}

Given a probability space $\{\Omega, F, P\}$, we consider the following semi-Markovian jump systems with time-varying delays:

$$
\left\{\begin{array}{l}
\dot{x}(t)=A\left(\lambda_{t}\right) x(t)+A_{d}\left(\lambda_{t}\right) x(t-\tau(t))+B\left(\lambda_{t}\right) \omega(t) \\
y(t)=C\left(\lambda_{t}\right) x(t)+C_{d}\left(\lambda_{t}\right) x(t-\tau(t))+D\left(\lambda_{t}\right) \omega(t) \\
z(t)=L\left(\lambda_{t}\right) x(t) \\
x(t)=\varphi(t), \quad t \in\left\{-\tau_{2}, \ldots, 0\right\}
\end{array}\right.
$$

where $x(t) \in \mathbb{R}^{n}$ is the system state vector; $y(t) \in \mathbb{R}^{p}$ is the measurement output; $z(t) \in \mathbb{R}^{q}$ is the control output; and $\omega(t) \in \mathbb{R}^{r}$ is the disturbance input, which belongs to $L_{2}[0, \infty) ; \varphi(t)$ is a initial condition. The process $\left\{\lambda_{t}\right\}$ is a time-homogeneous semi-Markov stochastic process, which takes value in a finite state space $\mathcal{S}=\{1,2, \ldots, s\}$. The evolution of $\lambda_{t}$ is governed by the following probability transition:

$$
\operatorname{Pr}\left\{\lambda_{t+h}=j \mid \lambda_{t}=i\right\}= \begin{cases}\phi_{i j}(h) h+o(h), & i \neq j, \\ 1+\phi_{i i}(h) h+o(h), & i=j\end{cases}
$$

where $h>0, \lim _{h \longrightarrow 0} 0(h) / h=0$, and $\phi_{i j}(h)$ is the transition rate from mode $i$ at time $t$ to mode $j$ at time $t+h$ when $i \neq j$ and

$$
\phi_{i i}(h)=-\sum_{j=1}^{s} \phi_{i j}(h)
$$

Remark 1. It is worth noting that when the transition rate $\phi_{i j}(h)$ of SMJSs is independent of $h$, that is, $\phi_{i j}(h)=\phi_{i j}$, then the SMJSs reduce to the common MJSs, which have been investigated extensively in $[1,2,18,40,41]$.

In system $(1), \tau(t)$ denotes the time-varying delay, which satisfies

$$
\tau_{1} \leq \tau(t) \leq \tau_{2}, \quad 0 \leq \dot{\tau}(t) \leq \mu<1 .
$$

To simplify the notation, for each $\lambda_{t}=i \in \mathcal{S}$, the matrices $A\left(\lambda_{t}\right), A_{d}\left(\lambda_{t}\right), B\left(\lambda_{t}\right), C\left(\lambda_{t}\right), C_{d}\left(\lambda_{t}\right), D\left(\lambda_{t}\right)$, and $L\left(\lambda_{t}\right)$ will be denoted by $A_{i}, A_{d i}, B_{i}, C_{i}, C_{d i}, D_{i}$, and $L_{i}$, respectively.

In this paper, the sampler is time-driven with a sampling period $g>0$. In the traditional method, the sampled data should be sent to the filter in every sampling period, and this would lead to need of more band resource. To solve this issue, we introduce an event-triggered processor between the sampler and filter to determine whether or not the current sampled data should be transmitted to the filter. It is assumed that the latest released output is $t_{k}$, then, the next released instant $t_{k+1}$ is determined by the following condition $[37,38]$ :

$$
\begin{aligned}
t_{k+1} & =\inf \left\{t>t_{k} \mid\left[y(t)-y\left(t_{k}\right)\right]^{T} \Upsilon_{i}\left[y(t)-y\left(t_{k}\right)\right]\right. \\
& \left.\geq \rho_{i} y(t)^{T} \Upsilon_{i} y(t)\right\}
\end{aligned}
$$

where $\rho_{i} \in(0,1)$ and $\Upsilon_{i}>0$ are event-triggered parameters.

For $t \in\left[t_{k}, t_{k+1}\right)$, we define the following function:

$$
\delta(t)=y(t)-y\left(t_{k}\right) .
$$

Then, by the event-triggered condition (5), we obtain

$$
\rho_{i} y(t)^{T} \Upsilon_{i} y(t)-\delta(t)^{T} \Upsilon_{i} \delta(t)>0 .
$$

This paper aims to design a filter as follows:

$$
\left\{\begin{array}{l}
\dot{x}_{f}(t)=A_{f}\left(\lambda_{t}\right) x_{f}(t)+B_{f}\left(\lambda_{t}\right) y\left(t_{k}\right), \\
z_{f}(t)=C_{f}\left(\lambda_{t}\right) x_{f}(t),
\end{array} \quad t \in\left[t_{k}, t_{k+1}\right),\right.
$$


where $x_{f}(t) \in \mathbb{R}^{n}$ is the filter state; $z_{f}(t) \in \mathbb{R}^{q}$ is the filter output; and $A_{f}\left(\lambda_{t}\right), B_{f}\left(\lambda_{t}\right)$, and $C_{f}\left(\lambda_{t}\right)$ are the filter parameters to be designed. For each $\lambda_{t}=i \in \mathcal{S}$, the matrices $A_{f}\left(\lambda_{t}\right), B_{f}\left(\lambda_{t}\right)$, and $C_{f}\left(\lambda_{t}\right)$ will be denoted by $A_{f i}, B_{f i}$, and $C_{f i}$.

Let $\xi(t)=\left[x(t) x_{f}(t)\right]^{T}$ and $e(t)=z(t)-z_{f}(t)$. Then, the filtering error system resulting from (1) and (8) is given by

$$
\left\{\begin{array}{l}
\dot{\xi}(t)=\widetilde{A}_{i} \xi(t)+\widetilde{A}_{d i} x(t-\tau(t))+\widetilde{B}_{\delta i}(t) \delta(t)+\widetilde{B}_{\omega i} \omega(t) \\
e(t)=\widetilde{L}_{i} \xi(t)
\end{array}\right.
$$

where

$$
\begin{aligned}
\widetilde{A}_{i} & =\left[\begin{array}{cc}
A_{i} & 0 \\
B_{f i} C_{i} & A_{f i}
\end{array}\right], \\
\widetilde{A}_{d i} & =\left[\begin{array}{c}
A_{d i} \\
B_{f i} C_{d i}
\end{array}\right], \\
\widetilde{B}_{\delta i} & =\left[\begin{array}{c}
0 \\
-B_{f i}
\end{array}\right], \\
\widetilde{B}_{\omega i} & =\left[\begin{array}{c}
B_{i} \\
B_{f i} D_{i}
\end{array}\right], \\
\widetilde{L}_{i} & =\left[\begin{array}{ll}
L_{i} & -C_{f i}
\end{array}\right] .
\end{aligned}
$$

Throughout this paper, the following definitions will be adopted.

Definition 1 (see [45]). System (1) with $\omega(t)=0$ is said to be stochastically stable if the following inequality holds for any initial mode $\lambda_{0}$ and initial condition $\varphi$ :

$$
\lim _{t \rightarrow \infty} \mathscr{E}\left\{\int_{0}^{t} x(t)^{T} x(t) \mathrm{d} t \mid x(0), \lambda_{0}\right\}<\infty .
$$

Definition 2 (see $[9,43]$ ). For given matrices $\mathscr{Z}, \mathscr{Y}, \mathscr{X}$ with $\mathscr{X} \leq 0$ and $\mathscr{X}$ is symmetric, the filtering error system (9) is said to be dissipative, if there exist $\theta>0$, such that for any $\mathscr{T}>0$ and zero initial condition, the following inequality holds:

$$
\mathscr{E}\left\{\int_{0}^{\mathscr{T}} J(t) \mathrm{d} t\right\} \geq \theta \mathscr{E}\left\{\int_{0}^{\mathscr{T}} \omega(t)^{T} \omega(t) \mathrm{d} t\right\},
$$

where $J(t)=e(t)^{T} \mathscr{Z} e(t)+2 e(t)^{T} \mathscr{Y} \omega(t)+\omega(t)^{T} \mathscr{X} \omega(t)$.

Noting that $\mathscr{Z} \leq 0$, we can deduce that there always exists matrix $\widetilde{\mathscr{Z}} \geq 0$, such that $\mathscr{Z}=-\widetilde{\mathscr{Z}}^{T} \widetilde{\mathscr{Z}}$.

The main purpose of this paper is to design a filter in the form of (8), such that the following conditions hold:

(1) When $\omega(t)=0$, the filtering error system (9) is stochastically stable

(2) When $\omega(t) \neq 0$ and $\omega(t) \in L_{2}[0, \infty)$, under zero initial condition, the filtering error system (9) is dissipative in the sense of Definition 2
To realize this purpose, we need to introduce the following lemmas.

Lemma 1 (see [44]). For any matrix $Z>0$, scalars $a, b$ with $b>a$, the following inequality holds:

$$
(b-a) \int_{a}^{b} \dot{x}(\alpha)^{T} Z \dot{x}(\alpha) \mathrm{d} \alpha \geq\left[\begin{array}{l}
\omega_{1} \\
\omega_{2}
\end{array}\right]^{T}\left[\begin{array}{cc}
Z & 0 \\
0 & 3 Z
\end{array}\right]\left[\begin{array}{l}
\omega_{1} \\
\omega_{2}
\end{array}\right],
$$

where $\omega_{1}=x(b)-x(a)$ and $\omega_{2}=x(b)+x(a)-2 /(b-a)$ $\int_{a}^{b} x(\alpha) d \alpha$.

The following lemma is a direct result of [45].

Lemma 2 (see [46]). For a given matrix $Z>0$, scalar $\varepsilon \in(0,1)$, then there exist matrices $Y_{1}$ and $Y_{2}$, such that the following inequality holds:

$$
\begin{aligned}
& {\left[\begin{array}{cc}
\frac{1}{\varepsilon} Z & 0 \\
0 & \frac{1}{1-\varepsilon} Z
\end{array}\right] \geq \operatorname{sym}\left\{Y_{1}\left[\begin{array}{ll}
I_{n} & 0_{n}
\end{array}\right]+Y_{2}\left[\begin{array}{ll}
0_{n} & I_{n}
\end{array}\right]\right\}} \\
& -\varepsilon Y_{1} Z^{-1} Y_{1}^{T}-(1-\varepsilon) Y_{2} Z^{-1} Y_{2}^{T} .
\end{aligned}
$$

Remark 2. It is well recognized that the reciprocally convex combination inequality in [47] is a powerful tool to handle the time-varying delays when analysing the stability of a system with time-varying delays. It has been shown that the inequality in Lemma 2 is a more accurate inequality than the reciprocally convex combination inequality; thus, the stability criteria derived by Lemma 2 is expected to be less conservative.

\section{Main Results}

In this section, we carry out the analysis of stochastic stability and dissipativity for the resulting filtering error system (9). Then, on the basis of these results, the desired filter design scheme for system (1) is established.

Theorem 1. For given scalars $\tau_{1}, \tau_{2}, \mu, 0<\rho_{i}<1$ and matrices $\mathscr{Z}, \mathcal{Y}, \mathscr{X}$ with $\mathscr{Z} \leq 0$ and $\mathscr{X}$ is symmetric, the filtering error system (9) is stochastically stable with dissipative, if there exist matrices $P_{i}>0, Q_{1 i}>0, Q_{2 i}>0, Q_{3 i}>0, S>0, R_{i}>0, U>0$, $Z_{i}>0, V>0$, and $Y_{i}>0$, any matrices $Y_{1}$ and $Y_{2}$, such that the following inequalities hold, for each $i \in \mathcal{S}$ :

$$
\begin{gathered}
\sum_{j=1}^{s} \phi_{i j}(h)\left(Q_{1 j}+Q_{3 j}\right)-S<0, \\
\sum_{j=1}^{s} \phi_{i j}(h)\left(Q_{2 j}+Q_{3 j}\right)-S<0,
\end{gathered}
$$




$$
\begin{gathered}
\sum_{j=1}^{s} \phi_{i j}(h) Q_{3 j}-S<0, \\
\tau_{1} \sum_{j=1}^{s} \phi_{i j}(h) R_{j}-U<0, \\
\tau_{12} \sum_{j=1}^{s} \phi_{i j}(h) Z_{j}-V<0, \\
{\left[\begin{array}{cc}
\Phi_{i}\left(\tau_{1}\right) & \Psi^{T} Y_{2} \\
* & -\widetilde{Z}_{i}
\end{array}\right]<0,} \\
{\left[\begin{array}{cc}
\Phi_{i}\left(\tau_{2}\right) & \Psi^{T} Y_{1} \\
* & -\widetilde{Z}_{i}
\end{array}\right]<0,}
\end{gathered}
$$

where

$$
\begin{aligned}
& \Phi_{i}(\tau(t))=W_{2 i}^{T} P_{i} W_{1}(\tau(t))+W_{1}(\tau(t))^{T} P_{i} W_{2 i} \\
& +W_{1}(\tau(t))^{T}\left(\sum_{j=1}^{s} \phi_{i j}(h) P_{j}\right) W_{1}(\tau(t)) \\
& +e_{1}^{T}\left(Q_{1 i}+Q_{2 i}+Q_{3 i}+\tau_{2} S\right) e_{1} \\
& -e_{3}^{T} Q_{1 i} e_{3}-(1-\mu) e_{4}^{T} Q_{2 i} e_{4} \\
& -e_{5}^{T} Q_{3 i} e_{5}+W_{s i}^{T}\left(\tau_{1}^{2} R_{i}+\frac{1}{2} \tau_{1}^{2} U+\tau_{12}^{2} Z_{i}+\frac{1}{2}\left(\tau_{2}^{2}-\tau_{1}^{2}\right) V\right) W_{s i} \\
& -\Psi^{T} \operatorname{sym}\left\{Y_{1}\left[I_{2 n} 0_{2 n}\right]+Y_{2}\left[0_{2 n} I_{2 n}\right]\right\} \Psi-W_{3}^{T} \widetilde{R}_{i} W_{3} \\
& +\rho_{i}\left(C_{i} e_{1}+C_{d i} e_{4}+D_{i} e_{10}\right)^{T} \\
& \cdot \Upsilon_{i}\left(C_{i} e_{1}+C_{d i} e_{4}+D_{i} e_{10}\right)-e_{9}^{T} \Upsilon_{i} e_{9} \\
& -W_{0}^{T} \widetilde{L}_{i}^{T} \mathscr{Z} \widetilde{L}_{i} W_{0}-2 W_{0}^{T} \widetilde{L}_{i}^{T} \mathscr{Y} e_{10}-e_{10}^{T} \mathscr{X} e_{10}, \\
& W_{s i}=\left[\begin{array}{lllll}
A_{i} & 0_{n, 2 n} & A_{d i} & 0_{n, 5 n} & B_{i}
\end{array}\right], \\
& \widetilde{W}_{s i}=\left[\begin{array}{llllll}
\widetilde{A}_{i} & 0_{2 n, n} & \widetilde{A}_{d i} & 0_{2 n, 4 n} & \widetilde{B}_{\delta i} & \widetilde{B}_{\omega i}
\end{array}\right], \\
& W_{0}=\operatorname{col}\left\{e_{1}, e_{2}\right\}, \quad \tau_{12}=\tau_{2}-\tau_{1}, \\
& W_{1}(\tau(t))=\operatorname{col}\left\{W_{0}, \tau_{1} e_{6},\left(\tau(t)-\tau_{1}\right) e_{7}-\left(\tau_{2}-\tau(t)\right) e_{8}\right\} \text {, } \\
& W_{2 i}=\operatorname{col}\left\{\tilde{W}_{s i}, e_{1}-e_{3}, e_{3}-e_{5}\right\}, \\
& W_{3}=\operatorname{col}\left\{e_{1}-e_{3}, e_{1}+e_{3}-2 e_{6}\right\}, \\
& W_{4}=\operatorname{col}\left\{e_{3}-e_{4}, e_{3}+e_{4}-2 e_{7}\right\} \text {, } \\
& W_{5}=\operatorname{col}\left\{e_{4}-e_{5}, e_{4}+e_{5}-2 e_{8}\right\} \text {, } \\
& e_{j}=\left[\begin{array}{lll}
0_{n,(j-1) n} & I_{n} & 0_{n,(8-j) n+p+r}
\end{array}\right], \quad j=1, \ldots, 8, \\
& e_{9}=\left[\begin{array}{lll}
0_{p, 8 n} & I_{p} & 0_{p, r}
\end{array}\right] \text {, } \\
& e_{10}=\left[\begin{array}{ll}
0_{r, 8 n+p} & I_{r}
\end{array}\right] \\
& \Psi=\operatorname{col}\left\{W_{4}, W_{5}\right\}, \\
& \widetilde{Z}_{i}=\operatorname{diag}\left(Z_{i}, 3 Z_{i}\right) \text {, } \\
& \widetilde{R}_{i}=\operatorname{diag}\left(R_{i}, 3 R_{i}\right) .
\end{aligned}
$$

Proof. We define the following augmented vector:

$$
\zeta(t)=\operatorname{col}\left\{\xi(t), \int_{t-\tau_{1}}^{t} x(\alpha) \mathrm{d} \alpha, \int_{t-\tau_{2}}^{t-\tau_{1}} x(\alpha) \mathrm{d} \alpha\right\}
$$

Then, we choose a Lyapunov-Krasovskii functional candidate for system (9) as follows:

$$
V\left(\xi_{t}, \lambda_{t}, t\right)=\sum_{j=1}^{4} V_{j}(t)
$$

where $\xi_{t}=\xi(t+\sigma), \sigma \in\left[-\tau_{2}, 0\right]$, and

$$
\begin{aligned}
V_{1}(t)= & \zeta(t)^{T} P_{i} \zeta(t), \\
V_{2}(t)= & \int_{t-\tau_{1}}^{t} x(\alpha)^{T} Q_{1 i} x(\alpha) \mathrm{d} \alpha+\int_{t-\tau(t)}^{t-\tau_{1}} x(\alpha)^{T} Q_{2 i} x(\alpha) \mathrm{d} \alpha \\
& +\int_{t-\tau_{2}}^{t} x(\alpha)^{T} Q_{3 i} x(\alpha) \mathrm{d} \alpha+\int_{-\tau_{2}}^{0} \int_{t+\beta}^{t} x(\alpha)^{T} S x(\alpha) \mathrm{d} \alpha, \\
V_{3}(t)= & \tau_{1} \int_{-\tau_{1}}^{0} \int_{t+\beta}^{t} \dot{x}(\alpha)^{T} R_{i} \dot{x}(\alpha) \mathrm{d} \alpha \mathrm{d} \beta \\
& +\int_{-\tau_{1}}^{0} \int_{\theta}^{0} \int_{t+\beta}^{t} \dot{x}(\alpha)^{T} U \dot{x}(\alpha) \mathrm{d} \alpha \mathrm{d} \beta \mathrm{d} \theta, \\
V_{4}(t)= & \tau_{12} \int_{-\tau_{2}}^{-\tau_{1}} \int_{t+\beta}^{t} \dot{x}(\alpha)^{T} Z_{i} \dot{x}(\alpha) \mathrm{d} \alpha \mathrm{d} \beta \\
& +\int_{-\tau_{2}}^{-\tau_{1}} \int_{\theta}^{0} \int_{t+\beta}^{t} \dot{x}(\alpha)^{T} V \dot{x}(\alpha) \mathrm{d} \alpha \mathrm{d} \beta \mathrm{d} \theta .
\end{aligned}
$$

Let $\mathscr{L}$ be the weak infinitesimal generator of the random process $\left\{\xi_{t}, \lambda_{t}\right\}$ and $\eta(t)=\mathrm{col}\left\{x(t) x_{f}(t), x\left(t-\tau_{1}\right), x(t-\right.$ $\tau(t)), x\left(t-\tau_{2}\right), 1 / \tau_{1} \int_{t-\tau_{1}}^{t} x(\alpha) \mathrm{d} \alpha, 1 /\left(\tau(t)-\tau_{1}\right) \int_{t-\tau(t)}^{t-\tau_{1}} x(\alpha)$ $\left.\mathrm{d} \alpha, 1 /\left(\tau_{2}-\tau(t)\right) \int_{t-\tau_{2}}^{t-\tau(t)} x(\alpha) \mathrm{d} \alpha, \delta(t), \omega(t)\right\}$. Then, for each $i \in \mathcal{S}$, following the similar lines as in $[13,27]$, we obtain

$$
\begin{aligned}
\mathscr{L} V_{1}(t)= & \dot{\zeta}(t)^{T} P_{i} \zeta(t)+\zeta(t)^{T} P_{i} \dot{\zeta}(t) \\
& +\zeta(t)^{T}\left(\sum_{j=1}^{s} \phi_{i j}(h) P_{j}\right) \zeta(t) \\
= & \eta(t)^{T}\left[W_{2 i}^{T} P_{i} W_{1}(\tau(t))+W_{1}(\tau(t))^{T} P_{i} W_{2 i}\right. \\
& \left.+W_{1}(\tau(t))^{T} \sum_{j=1}^{s} \phi_{i j}(h) P_{j} W_{1}(\tau(t))\right] \eta(t) .
\end{aligned}
$$

By means of (15)-(17), one has 


$$
\begin{aligned}
\mathscr{L} V_{2}(t) \leq & x(t)^{T}\left(Q_{1 i}+Q_{2 i}+Q_{3 i}+\tau_{2} S\right) x(t)-x\left(t-\tau_{1}\right)^{T} Q_{1 i} x\left(t-\tau_{1}\right) \\
& -(1-\mu) x(t-\tau(t))^{T} Q_{2 i} x(t-\tau(t))-x\left(t-\tau_{2}\right)^{T} Q_{3 i} x\left(t-\tau_{2}\right) \\
& +\int_{t-\tau_{1}}^{t} x(\alpha)^{T}\left(\sum_{j=1}^{s} \phi_{i j}(h) Q_{1 j}+\sum_{j=1}^{s} \phi_{i j}(h) Q_{3 j}-S\right) x(\alpha) \mathrm{d} \alpha \\
& +\int_{t-\tau(t)}^{t-\tau_{1}} x(\alpha)^{T}\left(\sum_{j=1}^{s} \phi_{i j}(h) Q_{2 j}+\sum_{j=1}^{s} \phi_{i j}(h) Q_{3 j}-S\right) x(\alpha) \mathrm{d} \alpha \\
& +\int_{t-\tau_{2}}^{t-\tau(t)} x(\alpha)^{T}\left(\sum_{j=1}^{s} \phi_{i j}(h) Q_{3 j}-S\right) x(\alpha) \mathrm{d} \alpha \\
\leq & \eta(t)^{T}\left[e_{1}^{T}\left(Q_{1 i}+Q_{2 i}+Q_{3 i}+\tau_{2} S\right) e_{1}-e_{3}^{T} Q_{1 i} e_{3}-(1-\mu) e_{4}^{T} Q_{2 i} e_{4}-e_{5}^{T} Q_{3 i} e_{5}\right] \eta(t) .
\end{aligned}
$$

In terms of (18), we deduce that

$$
\begin{aligned}
\mathscr{L} V_{3}(t)= & \tau_{1}^{2} \dot{x}(t)^{T} R_{i} \dot{x}(t)-\tau_{1} \int_{t-\tau_{1}}^{t} \dot{x}(\alpha)^{T} R_{i} \dot{x}(\alpha) \mathrm{d} \alpha \\
& +\tau_{1} \int_{-\tau_{1}}^{0} \int_{t+\beta}^{t} \dot{x}(\alpha)^{T} \sum_{j=1}^{s} \phi_{i j}(h) R_{j} \dot{x}(\alpha) \mathrm{d} \alpha \mathrm{d} \beta \\
& +\frac{1}{2} \tau_{1}^{2} \dot{x}(t)^{T} U \dot{x}(t)-\int_{-\tau_{1}}^{0} \int_{t+\beta}^{t} \dot{x}(\alpha)^{T} U \dot{x}(\alpha) \mathrm{d} \alpha \mathrm{d} \beta \\
\leq & \dot{x}(t)^{T}\left(\tau_{1}^{2} R_{i}+\frac{1}{2} \tau_{1}^{2} U\right) \dot{x}(t)-\tau_{1} \int_{t-\tau_{1}}^{t} \dot{x}(\alpha)^{T} R_{i} \dot{x}(\alpha) \mathrm{d} \alpha .
\end{aligned}
$$

$$
\begin{aligned}
-\tau_{1} \int_{t-\tau_{1}}^{t} \dot{x}(\alpha)^{T} R_{i} \dot{x}(\alpha) \mathrm{d} \alpha & \leq-\left[\begin{array}{c}
x(t)-x\left(t-\tau_{1}\right) \\
x(t)+x\left(t-\tau_{1}\right)-\frac{2}{\tau_{1}} \int_{t-\tau_{1}}^{t} x(\alpha) \mathrm{d} \alpha
\end{array}\right]^{T}\left[\begin{array}{cc}
R_{i} & 0 \\
0 & 3 R_{i}
\end{array}\right] \times\left[\begin{array}{c}
x(t)-x\left(t-\tau_{1}\right) \\
x(t)+x\left(t-\tau_{1}\right)-\frac{2}{\tau_{1}} \int_{t-\tau_{1}}^{t} x(\alpha) \mathrm{d} \alpha
\end{array}\right] \\
& =-\eta(t)^{T}\left[\begin{array}{c}
e_{1}-e_{3} \\
e_{1}+e_{3}-2 e_{6}
\end{array}\right]^{T} \widetilde{R}_{i}\left[\begin{array}{c}
e_{1}-e_{3} \\
e_{1}+e_{3}-2 e_{6}
\end{array}\right] \eta(t) \\
& =-\eta(t)^{T} W_{3}^{T} \widetilde{R}_{i} W_{3} \eta(t) .
\end{aligned}
$$

Then, it follows from (28) and (29) that Now, $\mathscr{L} V_{4}(t)$ is obtained as $\mathscr{L} V_{3}(t) \leq \eta(t)^{T}\left[W_{s i}^{T}\left(\tau_{1}^{2} R_{i}+\frac{1}{2} \tau_{1}^{2} U\right) W_{s i}-W_{3}^{T} \widetilde{R}_{i} W_{3}\right] \eta(t)$.

$$
\begin{aligned}
\mathscr{L} V_{4}(t)= & \tau_{12}^{2} \dot{x}(t)^{T} Z_{i} \dot{x}(t)-\tau_{12} \int_{t-\tau_{2}}^{t-\tau_{1}} \dot{x}(\alpha)^{T} Z_{i} \dot{x}(\alpha) \mathrm{d} \alpha+\tau_{12} \int_{-\tau_{2}}^{-\tau_{1}} \int_{t+\beta}^{t} \dot{x}(\alpha)^{T}\left(\sum_{j=1}^{s} \phi_{i j}(h) Z_{j}\right) \dot{x}(\alpha) \mathrm{d} \alpha \mathrm{d} \beta \\
& +\frac{1}{2}\left(\tau_{2}^{2}-\tau_{1}^{2}\right) \dot{x}(t)^{T} V \dot{x}(t)-\int_{-\tau_{2}}^{-\tau_{1}} \int_{t+\beta}^{t} \dot{x}(\alpha)^{T} V \dot{x}(\alpha) \mathrm{d} \alpha \mathrm{d} \beta .
\end{aligned}
$$


Splitting the following integral into two terms, we obtain By Lemma 1, it can be verified that

$$
\begin{aligned}
-\tau_{12} \int_{t-\tau_{2}}^{t-\tau_{1}} \dot{x}(\alpha)^{T} Z_{i} \dot{x}(\alpha) \mathrm{d} \alpha= & -\tau_{12} \int_{t-\tau(t)}^{t-\tau_{1}} \dot{x}(\alpha)^{T} Z_{i} \dot{x}(\alpha) \mathrm{d} \alpha \\
& -\tau_{12} \int_{t-\tau_{2}}^{t-\tau(t)} \dot{x}(\alpha)^{T} Z_{i} \dot{x}(\alpha) \mathrm{d} \alpha .
\end{aligned}
$$

$$
\begin{aligned}
& -\tau_{12} \int_{t-\tau(t)}^{t-\tau_{1}} \dot{x}(\alpha)^{T} Z_{i} \dot{x}(\alpha) \mathrm{d} \alpha \\
& \leq-\frac{\tau_{12}}{\tau(t)-\tau_{1}}\left[\begin{array}{c}
x\left(t-\tau_{1}\right)-x(t-\tau(t)) \\
x\left(t-\tau_{1}\right)+x(t-\tau(t))-\frac{2}{\left(\tau(t)-\tau_{1}\right)} \int_{t-\tau(t)}^{t-\tau_{1}} x(\alpha) \mathrm{d} \alpha
\end{array}\right]\left[\begin{array}{cc}
Z_{i} & 0 \\
0 & 3 Z_{i}
\end{array}\right] \\
& \times\left[\begin{array}{c}
x\left(t-\tau_{1}\right)-x(t-\tau(t)) \\
x\left(t-\tau_{1}\right)+x(t-\tau(t))-\frac{2}{\left(\tau(t)-\tau_{1}\right)} \int_{t-\tau(t)}^{t-\tau_{1}} x(\alpha) \mathrm{d} \alpha
\end{array}\right] \\
& =-\frac{\tau_{12}}{\tau(t)-\tau_{1}} \eta(t)^{T}\left[\begin{array}{c}
e_{3}-e_{4} \\
e_{3}+e_{4}-2 e_{7}
\end{array}\right]^{T} \widetilde{Z}_{i}\left[\begin{array}{c}
e_{3}-e_{4} \\
e_{3}+e_{4}-2 e_{7}
\end{array}\right] \eta(t) \\
& =-\frac{\tau_{12}}{\tau(t)-\tau_{1}} \eta(t)^{T} W_{4}^{T} \widetilde{Z}_{i} W_{4} \eta(t), \\
& -\tau_{12} \int_{t-\tau_{2}}^{t-\tau(t)} \dot{x}(\alpha)^{T} Z_{i} \dot{x}(\alpha) \mathrm{d} \alpha
\end{aligned}
$$

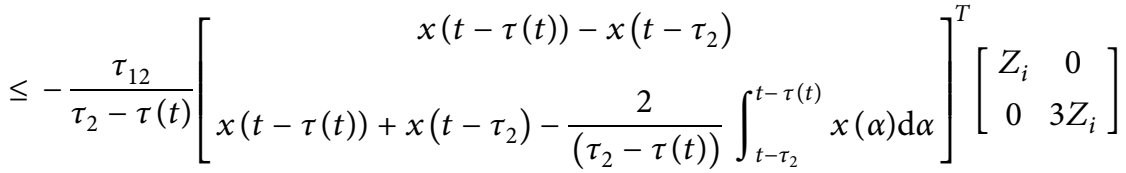

$$
\begin{aligned}
& \times\left[\begin{array}{c}
x(t-\tau(t))-x\left(t-\tau_{2}\right) \\
x(t-\tau(t))+x\left(t-\tau_{2}\right)-\frac{2}{\left(\tau_{2}-\tau(t)\right)} \int_{t-\tau_{2}}^{t-\tau(t)} x(\alpha) \mathrm{d} \alpha
\end{array}\right] \\
& =-\frac{\tau_{12}}{\tau_{2}-\tau(t)} \eta(t)^{T}\left[\begin{array}{c}
e_{4}-e_{5} \\
e_{4}+e_{5}-2 e_{8}
\end{array}\right]^{T} \widetilde{Z}_{i}\left[\begin{array}{c}
e_{4}-e_{5} \\
e_{4}+e_{4}-2 e_{8}
\end{array}\right] \eta(t) \\
& =-\frac{\tau_{12}}{\tau_{2}-\tau(t)} \eta(t)^{T} W_{5}^{T} \widetilde{Z}_{i} W_{5} \eta(t) \text {. }
\end{aligned}
$$


In light of (33)-(34) and Lemma 2, there exist matrices $Y_{1}$ and $Y_{2}$, such that

$$
\begin{aligned}
& -\tau_{12} \int_{t-\tau_{2}}^{t-\tau_{1}} \dot{x}(\alpha)^{T} Z_{i} \dot{x}(\alpha) \mathrm{d} \alpha \\
\leq & -\frac{\tau_{12}}{\tau(t)-\tau_{1}} \eta(t)^{T} W_{4}^{T} \widetilde{Z}_{i} W_{4} \eta(t) \\
& -\frac{\tau_{12}}{\tau_{2}-\tau(t)} \eta(t)^{T} W_{5}^{T} \widetilde{Z}_{i} W_{5} \eta(t) \\
\leq & -\eta(t)^{T} \Psi^{T} \operatorname{sym}\left\{Y_{1}\left[I_{2 n} 0_{2 n}\right]+Y_{2}\left[0_{2 n} I_{2 n}\right]\right\} \Psi \eta(t) \\
& +\frac{\tau(t)-\tau_{1}}{\tau_{12}} \eta(t)^{T} \Psi^{T} Y_{1} \widetilde{Z}_{i}^{-1} Y_{1}^{T} \Psi \eta(t) \\
& +\frac{\tau_{2}-\tau(t)}{\tau_{12}} \eta(t)^{T} \Psi^{T} Y_{2} \widetilde{Z}_{i}^{-1} Y_{2}^{T} \Psi \eta(t) .
\end{aligned}
$$

Then, it follows from (31)-(35) together with (19) that

$$
\begin{aligned}
\mathscr{L} V_{4}(t) \leq & \dot{x}(t)^{T}\left[\tau_{12}^{2} Z_{i}+\frac{1}{2}\left(\tau_{2}^{2}-\tau_{1}^{2}\right) V\right] \dot{x}(t) \\
& -\tau_{12} \int_{t-\tau_{2}}^{t-\tau_{1}} \dot{x}(\alpha)^{T} Z_{i} \dot{x}(\alpha) \mathrm{d} \alpha \\
\leq & \eta(t)^{T}\left[W_{s i}^{T}\left(\tau_{12}^{2} Z_{i}+\frac{1}{2}\left(\tau_{2}^{2}-\tau_{1}^{2}\right) V\right) W_{s i}\right] \eta(t) \\
& -\eta(t)^{T} \Psi^{T} \operatorname{sym}\left\{Y_{1}\left[I_{2 n} 0_{2 n}\right]+Y_{2}\left[0_{2 n} I_{2 n}\right]\right\} \Psi \eta(t) \\
& +\frac{\tau(t)-\tau_{1}}{\tau_{12}} \eta(t)^{T} \Psi^{T} Y_{1} \widetilde{Z}_{i}^{-1} Y_{1}^{T} \Psi \eta(t) \\
& +\frac{\tau_{2}-\tau(t)}{\tau_{12}} \eta(t)^{T} \Psi^{T} Y_{2} \widetilde{Z}_{i}^{-1} Y_{2}^{T} \Psi \eta(t) .
\end{aligned}
$$

Then, by (24)-(27), (30), and (36), we arrive at

$$
\begin{aligned}
& \mathscr{L} V(t)-J(t)+\rho_{i} y(t)^{T} \Upsilon_{i} y(t)-\delta(t)^{T} \Upsilon_{i} \delta(t) \\
& \quad \leq \eta(t)^{T} \widetilde{\Phi}_{i}(\tau(t)) \eta(t),
\end{aligned}
$$

where

$$
\begin{aligned}
\widetilde{\Phi}_{i}(\tau(t))= & \Phi_{i}(\tau(t))+\frac{\tau(t)-\tau_{1}}{\tau_{12}} \Psi^{T} Y_{1} \widetilde{Z}_{i}^{-1} Y_{1}^{T} \Psi \\
& +\frac{\tau_{2}-\tau(t)}{\tau_{12}} \Psi^{T} Y_{2} \widetilde{Z}_{i}^{-1} Y_{2}^{T} \Psi
\end{aligned}
$$

and $\Phi_{i}(\tau(t))$ is defined in Theorem 1 .

By convex combination theory, it can be deduced that $\widetilde{\Phi}_{i}(\tau(t))<0$ holds if $\widetilde{\Phi}_{i}\left(\tau_{1}\right)<0$ and $\widetilde{\Phi}_{i}\left(\tau_{2}\right)<0$. These two inequalities can be obtained by applying Schur complement to (20) and (21), respectively.

Therefore, inequalities (7) and (37) lead to

$$
\mathscr{L} V(t)-J(t)<0
$$

Using the well-known Dykin's formula to (39), we obtain that, for any $\mathscr{T} \geq 0$,

$$
\begin{aligned}
& \mathscr{E}\left\{\int_{0}^{\mathscr{T}} J(t) \mathrm{d} t\right\}>\mathscr{E}\left\{V\left(\xi_{t}, \lambda_{t}, t\right)\right\} \\
& -V\left(\xi_{0}, \lambda_{0}, 0\right)>0 .
\end{aligned}
$$

Let $\mathscr{T} \longrightarrow \infty$. It follows from (40) that there exists $\theta>0$, such that inequality (12) holds. Thus, we conclude that the filtering error system (9) is dissipative in the sense of Definition 2.

Next, we will prove that the filtering error system (9) is stochastically stable. Let $\omega(t)=0$, then (39) leads to

$$
\mathscr{L} V(t)<e(t)^{T} \mathscr{Z} e(t)
$$

Noticing that $\mathscr{Z} \leq 0$, we obtain that there exists a sufficient small $\varsigma>0$, such that $\mathscr{L} V(t)<-\varsigma|x(t)|^{2}$. Then, along a similar line as in the proof of [41], we derive that the filtering error system (9) is stochastically stable. The proof is complete here.

Remark 3. Theorem 1 provides a sufficient condition such that the filtering error system (9) is stochastically stable and dissipative. It is noted that this condition is dependent not only on system modes and time delays but also on eventtriggered parameters.

In order to compare with previous results, we consider the case when system (1) with $\omega(t)=0$ :

$$
\dot{x}(t)=A_{i} x(t)+A_{d i} x(t-\tau(t)) .
$$

The stochastic stability condition of system (42) can be derived from Theorem 1 immediately. We give the result in the following corollary.

Corollary 1. For given scalars $\tau_{1}, \tau_{2}$, and $\mu$, system (42) is stochastically stable if there exist matrices $P_{i}>0, Q_{1 i}>0$, $Q_{2 i}>0, Q_{3 i}>0, S>0, R_{i}>0, U>0, Z_{i}>0, V>0$, and any matrices $Y_{1}$ and $Y_{2}$, such that inequalities (15)-(19) and the following inequalities hold, for each $i \in \mathcal{S}$ : 


$$
\begin{aligned}
& {\left[\begin{array}{cc}
\Xi_{i}\left(\tau_{1}\right) & \widetilde{\Psi}^{T} Y_{2} \\
* & -\widetilde{Z}_{i}
\end{array}\right]<0,} \\
& {\left[\begin{array}{cc}
\Xi_{i}\left(\tau_{2}\right) & \widetilde{\Psi}^{T} Y_{1} \\
* & -\widetilde{Z}_{i}
\end{array}\right]<0,}
\end{aligned}
$$

where

$$
\begin{aligned}
\Xi_{i}(\tau(t))= & E_{2 i}^{T} P_{i} E_{1}(\tau(t))+E_{1}(\tau(t))^{T} P_{i} E_{2 i}+E_{1}(\tau(t))^{T}\left(\sum_{j=1}^{s} \phi_{i j}(h) P_{j}\right) E_{1}(\tau(t)) \\
& +\widetilde{e}_{1}^{T}\left(Q_{1 i}+Q_{2 i}+Q_{3 i}+\tau_{2} S\right) \widetilde{e}_{1}-\widetilde{e}_{2}^{T} Q_{1 i} \widetilde{e}_{2}-(1-\mu) \widetilde{e}_{3}^{T} Q_{2 i} \widetilde{e}_{3}-\widetilde{e}_{4}^{T} Q_{3 i} \widetilde{e}_{4} \\
& +\left(A_{i} \widetilde{e}_{1}+A_{d i} \widetilde{e}_{3}\right)^{T}\left(\tau_{1}^{2} R_{i}+\frac{1}{2} \tau_{1}^{2} U+\tau_{12}^{2} Z_{i}+\frac{1}{2}\left(\tau_{2}^{2}-\tau_{1}^{2}\right) V\right)\left(A_{i} \widetilde{e}_{1}+A_{d i} \widetilde{e}_{3}\right) \\
& -E_{3}^{T} \widetilde{R}_{i} E_{3}-\widetilde{\Psi}^{T} \operatorname{sym}\left\{Y_{1}\left[I_{2 n} 0_{2 n}\right]+Y_{2}\left[0_{2 n} I_{2 n}\right]\right\} \widetilde{\Psi}, \\
\widetilde{e}_{i}= & \left(00_{n,(i-1) n} I_{n} \quad 0_{n,(7-i) n}\right), \quad i=1,2, \ldots, 7, \\
E_{1}(\tau(t))= & \operatorname{col}\left\{\widetilde{e}_{1}, \tau_{1} \widetilde{e}_{5},\left(\tau(t)-\tau_{1}\right) \widetilde{e}_{6}+\left(\tau_{2}-\tau(t)\right) \widetilde{e}_{7}\right\}, \\
E_{2 i}= & \operatorname{col}\left\{A_{i} \widetilde{e}_{1}+A_{d i} \widetilde{e}_{3}, \widetilde{e}_{1}-\widetilde{e}_{2}, \widetilde{e}_{2}-\widetilde{e}_{4}\right\}, \\
\widetilde{\Psi}_{3}= & \operatorname{col}\left\{E_{4}, E_{5}\right\} . \\
E_{3}= & \operatorname{col}\left\{\widetilde{e}_{1}-\widetilde{e}_{2}, \widetilde{e}_{1}+\widetilde{e}_{2}-2 \widetilde{e}_{5}\right\}, \\
E_{4}= & \operatorname{col}\left\{\widetilde{e}_{2}-\widetilde{e}_{3}, \widetilde{e}_{2}+\widetilde{e}_{3}-2 \widetilde{e}_{6}\right\}, \\
& \operatorname{col}\left\{\widetilde{e}_{3}-\widetilde{e}_{4}, \widetilde{e}_{3}+\widetilde{e}_{4}-2 \widetilde{e}_{7}\right\},
\end{aligned}
$$

Remark 4. Corollary 1 provides a new stochastic stability criterion for SMJSs (42). It is important to note that the condition in Corollary 1 is less conservative than some of the existing ones in the literature, which will be shown by a numerical example in Section 4.

Based on Theorem 1, we give the filter parameter design scheme in the following theorem:

Theorem 2. For given scalars $\tau_{1}, \tau_{2}$, and $\mu, 0<\rho_{i}<1$, the filtering error system (9) is stochastically stable with dissipativity, if there exist matrices $Q_{1 i}>0, Q_{2 i}>0, Q_{3 i}>0, S>0$, $R_{i}>0, U>0, Z_{i}>0, V>0, M_{i}>0, X_{i}>0, P_{2 i}>0, P_{3 i}>0$, and $\Upsilon_{i}>0$, any matrices $Y_{1}, Y_{2}, \bar{A}_{f i}, \bar{B}_{f i}$, and $\bar{C}_{f i}$, such that inequalities (15)-(19) and the following inequalities hold, for each $i \in \mathcal{S}$ :

$$
\begin{aligned}
& {\left[\begin{array}{ll}
M_{i} & X_{i} \\
X_{i} & X_{i}
\end{array}\right]>0,} \\
& {\left[\begin{array}{ccc}
\widehat{\Phi}_{i}\left(\tau_{1}\right) & \Psi^{T} Y_{2} & W_{0}^{T} \widetilde{L}_{i}^{T} \widetilde{\mathscr{Z}}^{T} \\
* & -\widetilde{Z}_{i} & 0 \\
* & * & -I
\end{array}\right]<0,} \\
& {\left[\begin{array}{ccc}
\widehat{\Phi}_{i}\left(\tau_{2}\right) & \Psi^{T} Y_{1} & W_{0}^{T} \widetilde{L}_{i}^{T} \widetilde{\mathscr{Z}}^{T} \\
* & -\widetilde{Z}_{i} & 0 \\
* & * & -I
\end{array}\right]<0,}
\end{aligned}
$$

where 


$$
\begin{aligned}
& \widehat{\Phi}_{i}(\tau(t))=\widehat{W}_{2 i}^{T} W_{1}(\tau(t))+W_{1}(\tau(t))^{T} \widehat{W}_{2 i}+W_{1}(\tau(t))^{T}\left(\sum_{j=1}^{s} \phi_{i j}(h) P_{j}\right) W_{1}(\tau(t)) \\
& +e_{1}^{T}\left(Q_{1 i}+Q_{2 i}+Q_{3 i}+\tau_{2} S\right) e_{1}-e_{3}^{T} Q_{1 i} e_{3}-(1-\mu) e_{4}^{T} Q_{2 i} e_{4} \\
& -e_{5}^{T} Q_{3 i} e_{5}+W_{s i}^{T}\left(\tau_{1}^{2} R_{i}+\frac{1}{2} \tau_{1}^{2} U+\tau_{12}^{2} Z_{i}+\frac{1}{2}\left(\tau_{2}^{2}-\tau_{1}^{2}\right) V\right) W_{s i} \\
& -\Psi^{T} \operatorname{sym}\left\{Y_{1}\left[I_{2 n} 0_{2 n}\right]+Y_{2}\left[0_{2 n} I_{2 n}\right]\right\} \Psi-W_{3}^{T} \widetilde{R}_{i} W_{3} \\
& +\rho_{i}\left(C_{i} e_{1}+C_{d i} e_{4}+D_{i} e_{10}\right)^{T} \Upsilon_{i}\left(C_{i} e_{1}+C_{d i} e_{4}+D_{i} e_{10}\right)-e_{9}^{T} \Upsilon_{i} e_{9} \\
& -2 W_{0}^{T} \widetilde{L}_{i}^{T} \mathscr{Y} e_{10}-e_{10}^{T} \mathscr{X} e_{10} \\
& \widehat{W}_{2 i}=\operatorname{col}\left\{\widehat{W}_{s i}, P_{2 i}\left(e_{1}-e_{3}\right), P_{3 i}\left(e_{3}-e_{5}\right)\right\}, \\
& \widehat{W}_{s i}=\operatorname{col}\left\{\widehat{A}_{i}, 0_{2 n, n}, \widehat{A}_{d i}, 0_{2 n, 4 n}, \widehat{B}_{\delta i}, \widehat{B}_{\omega i}\right\} \\
& \widehat{A}_{i}=\left[\begin{array}{cc}
M_{i} A_{i}+\bar{B}_{f i} C_{i} & \bar{A}_{f i} \\
X_{i} A_{i}+\bar{B}_{f i} C_{i} & \bar{A}_{f i}
\end{array}\right], \\
& \widehat{A}_{d i}=\left[\begin{array}{c}
M_{i} A_{d i}+\bar{B}_{f i} C_{d i} \\
X_{i} A_{d i}+\bar{B}_{f i} C_{d i}
\end{array}\right], \\
& \widehat{B}_{\delta i}=\left[\begin{array}{c}
-\bar{B}_{f i} \\
-\bar{B}_{f i}
\end{array}\right] \\
& \widehat{B}_{\omega i}=\left[\begin{array}{c}
M_{i} B_{i}+\bar{B}_{f i} D_{i} \\
X_{i} B_{i}+\bar{B}_{f i} D_{i}
\end{array}\right] .
\end{aligned}
$$

In this case, a desired filter is obtained with the parameters given by

$$
\begin{aligned}
& A_{f i}=X_{i}^{-1} \bar{A}_{f i}, \\
& B_{f i}=X_{i}^{-1} \bar{B}_{f i}, \\
& C_{f i}=\bar{C}_{f i} .
\end{aligned}
$$

Proof. Define $P_{1 i}=\left[\begin{array}{cc}M_{i} & X_{i} \\ X_{i} & X_{i}\end{array}\right]$ and $P_{i}=\operatorname{diag}\left(P_{1 i}, P_{2 i}, P_{3 i}\right)$. It can be seen from (45) that $P_{i}>0$.
Let $\bar{A}_{f i}=X_{i} A_{f i}, \bar{B}_{f i}=X_{i} B_{f i}$, and $\bar{C}_{f i}=C_{f i}$. Then, substituting those matrices and $P_{i}$ into (46)-(47), we obtain (20) and (21). Therefore, according to Theorem 1, we easily draw the conclusion that the filtering error system (9) is stochastically stable and dissipative; thus, the proof is completed.

Remark 5. It should be pointed out that the results given in Theorem 1 are nonconvex inequalities, so it should be further discussed. Motivated by [13, 27], the transition rate $\phi_{i j}(h)$ is usually partly measurable in practice while it satisfies $\phi_{i j} \leq \phi_{i j}(h) \leq \bar{\phi}_{i j}$. Then, the $\phi_{i j}(h)$ can be rewritten as the following: 


$$
\begin{aligned}
\phi_{i j}(h)= & \sum_{\alpha=1}^{N} \psi_{\alpha} \phi_{i j, \alpha}, \quad \sum_{\alpha=1}^{N} \psi_{\alpha}=1, \psi_{\alpha} \geq 0, \\
\phi_{i j, \alpha}= & \begin{array}{ll}
\underline{\phi}_{i j}+(\alpha-1) \frac{\bar{\phi}_{i j}-\underline{\phi}_{i j}}{N-1}, & i \neq j, j \in \mathcal{S}, \\
\bar{\phi}_{i j}-(\alpha-1) \frac{\bar{\phi}_{i j}-\underline{\phi}_{i j}}{N-1}, & i=j, j \in \mathcal{S} .
\end{array}
\end{aligned}
$$

Now, we give the conditions for the solvability of the present dissipative filter design problem.

Theorem 3. For given scalars $\tau_{1}, \tau_{2}, \mu$, and $\rho_{i}$, the filtering error system (9) is stochastically stable with dissipativity, if there exist matrices $Q_{1 i}>0, Q_{2 i}>0, Q_{3 i}>0, S>0, R_{i}>0$, $U>0, Z_{i}>0, V>0, M_{i}>0, X_{i}>0, P_{2 i}>0, P_{3 i}>0$, and $Y_{i}>0$, any matrices $Y_{1}, Y_{2}, \bar{A}_{f i}, \bar{B}_{f i}$, and $\bar{C}_{f i}$, such that inequalities (15)-(19) and (45)-(47) hold, for each $i \in \mathcal{S}$, and $\phi_{i j}(h)=\phi_{i j, \alpha}, \alpha=1,2, \ldots, N$. Then, the desired filter $p a-$ rameters can be shown as in (49).

Remark 6. It is noted that the conditions obtained in Theorem 3 are presented in terms of linear matrix inequalities, which can be checked easily by the LMI toolbox of MATLAB.

\section{Numerical Examples}

Example 1. Consider a semi-Markovian jumping system in (42) with the following parameters [28]:

$$
\begin{aligned}
& A_{1}=\left[\begin{array}{cc}
-2 & 0 \\
0 & -0.9
\end{array}\right], \\
& A_{2}=\left[\begin{array}{ll}
-1 & 0.5 \\
0 & -1
\end{array}\right], \\
& A_{d 1}=\left[\begin{array}{cc}
-1 & 0 \\
-1 & -1
\end{array}\right], \\
& A_{d 2}=\left[\begin{array}{cc}
-1 & 0 \\
-0.1 & -1
\end{array}\right] .
\end{aligned}
$$

To compare the stability criterion in Corollary 1 with those in $[28,41,48,49]$, we assume that $\tau_{1}=1, \mu=0.5$, $\phi_{11}=-0.1$, and $\phi_{22}=-0.8$. Table 1 lists the maximum allowed $\tau_{2}$ calculated by the method in [28, 41, 48, 49] and Corollary 1 . It can be seen from Table 1 that the result in Corollary 1 is less conservative than those in $[28,41,48,49]$
TABLE 1: Maximum allowable $\tau_{2}$ using different methods.

\begin{tabular}{lc}
\hline Methods & $\tau_{2}$ \\
\hline Theorem 1 of [41] & 1.471 \\
Theorem 1 of [48] & 1.660 \\
Theorem 1 of [49] $(m=5)$ & 1.753 \\
Theorem 1 of [28] $(l=4)$ & 1.807 \\
Corollary 1 & 1.982 \\
\hline
\end{tabular}

for this example. It should be noted that the reduced conservatism in Corollary 1 benefits from using the inequalities in Lemma 1 and Lemma 2.

Example 2. Consider the semi-Markovian jumping system in (1) with the following parameters:

$$
\left.\begin{array}{rl}
A_{1} & =\left[\begin{array}{ll}
-3.4888 & 0.8057 \\
-0.6451 & -3.2684
\end{array}\right], \\
A_{2} & =\left[\begin{array}{ll}
-2.4898 & 0.2895 \\
1.3396 & -0.0211
\end{array}\right], \\
A_{d 1} & =\left[\begin{array}{ll}
-0.8620 & -1.2919 \\
-0.6841 & -2.0729
\end{array}\right], \\
A_{d 2} & =\left[\begin{array}{ll}
-2.8306 & 0.4978 \\
-0.8436 & -1.0115
\end{array}\right], \\
B_{1} & =\left[\begin{array}{ll}
0.0403 \\
0.6771
\end{array}\right], \\
D_{1} & =\left[\begin{array}{ll}
0.5689 \\
B_{2}
\end{array}\right], \\
D_{1} & =\left[\begin{array}{ll}
-1.3324 & -1.5532
\end{array}\right], \\
L_{2} & =\left[\begin{array}{ll}
-1.4562 & -1.2322
\end{array}\right], \\
C_{1} & =\left[\begin{array}{ll}
-0.3775 & -0.2959
\end{array}\right], \\
C_{2} & =\left[\begin{array}{ll}
-0.4751 & -0.2340
\end{array}\right], \\
C_{d 1} & =\left[\begin{array}{ll}
0.2532 & -0.1684
\end{array}\right], \\
D_{d 2} & -0.3564
\end{array}\right],
$$

In this example, the parameters are given by $\tau_{1}=0.1$, $\tau_{2}=0.5, \quad \phi_{11}(h) \in(-2.2,-1.8), \quad \phi_{22}(h) \in(-3.4,-2.6)$, $\rho_{1}=0.1, \rho_{2}=0.2, \mathscr{Z}=-4, \mathscr{Y}=-1$, and $\mathscr{X}=2$. Then, by solving the LMIs in Theorem 3, we obtain the dissipative filter parameters as follows: 


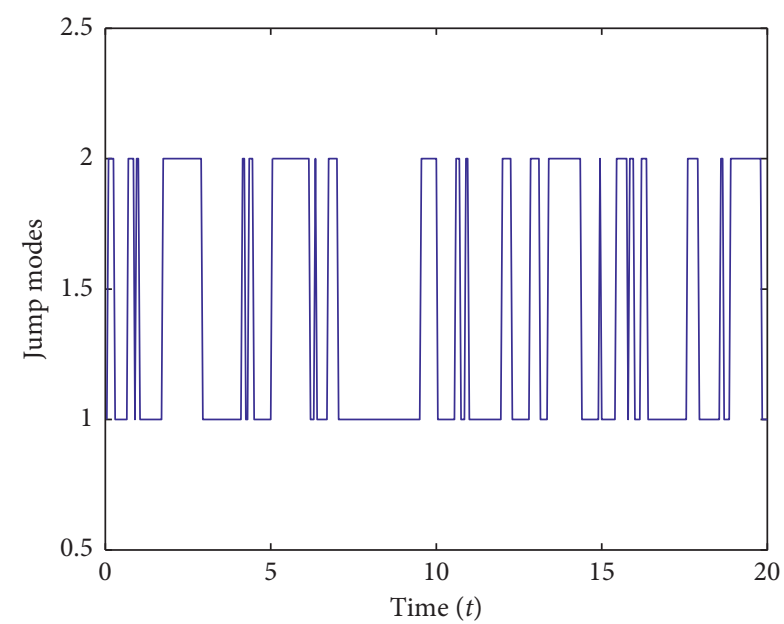

Figure 1: Random jump modes $\lambda_{t}$.

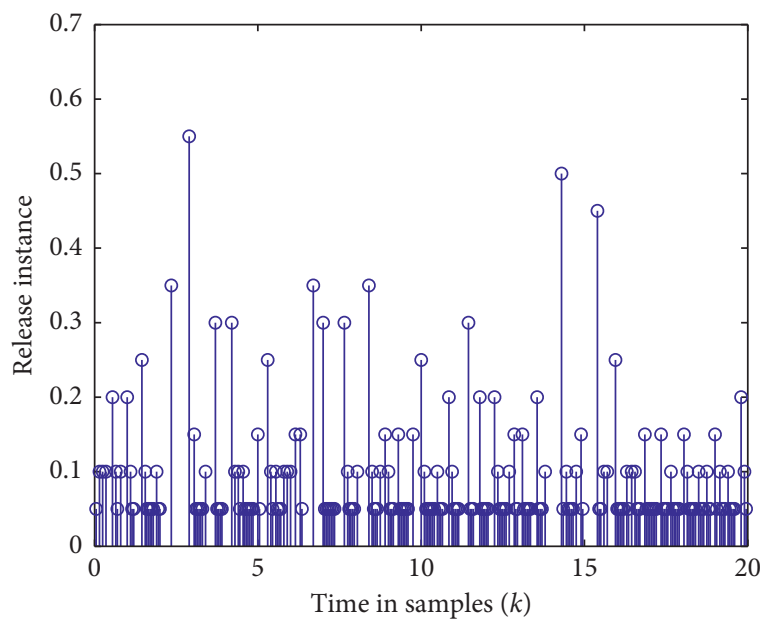

FIgURE 2: Release instants and release interval.

$$
\begin{aligned}
A_{f 1} & =\left[\begin{array}{ll}
-6.8697 & 0.8567 \\
-3.1332 & -6.4577
\end{array}\right], \\
B_{f 1} & =\left[\begin{array}{c}
-0.4229 \\
0.4993
\end{array}\right], \\
C_{f 1} & =\left[\begin{array}{l}
1.1557 \\
0.8781
\end{array}\right], \\
A_{f 2} & =\left[\begin{array}{l}
-5.8309 \\
1.3407
\end{array}\right]-1.4748 \\
B_{f 2} & =\left[\begin{array}{l}
3.4855 \\
0.6502
\end{array}\right], \\
C_{f 1} & =\left[\begin{array}{l}
0.6011 \\
0.9456
\end{array}\right]^{T}, \\
\Upsilon_{1} & =4.8762 \\
\Upsilon_{2} & =13.9442 .
\end{aligned}
$$

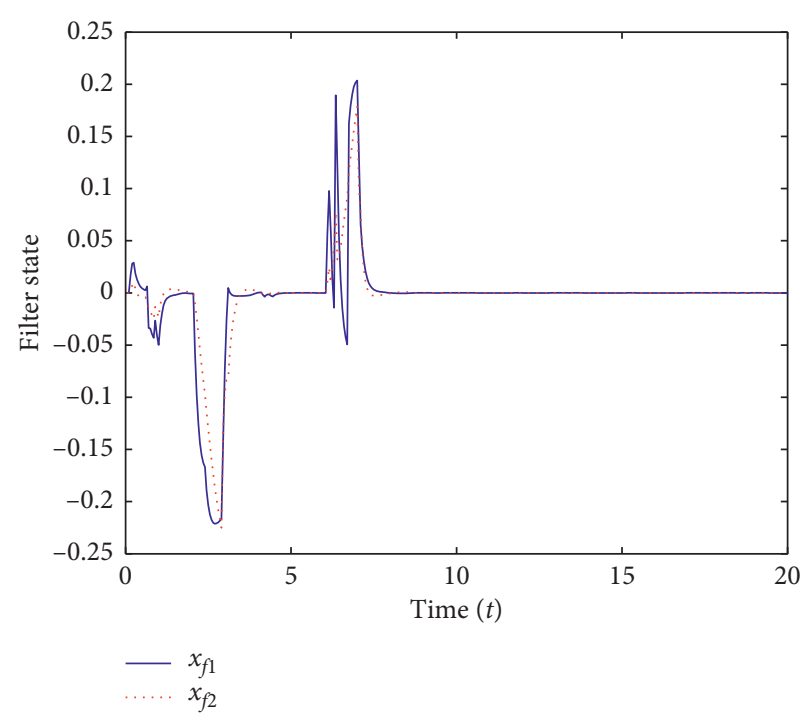

Figure 3: Filter states $x_{f}(t)$.

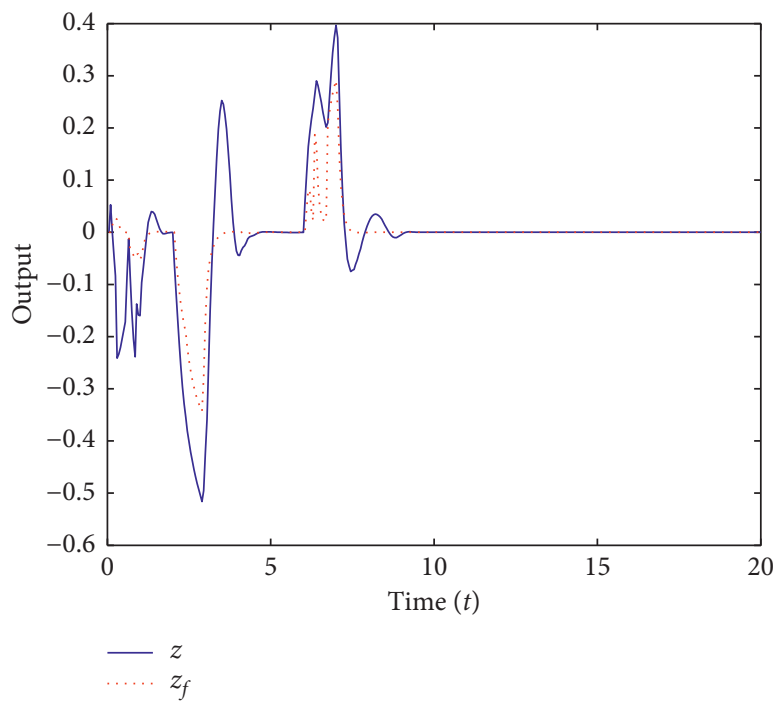

FIGURE 4: Estimated signal $z(t)$ and filter output signal $z_{f}(t)$.

In the simulation, we choose the initial condition $x(0)=$ $\left[\begin{array}{ll}-1 & 1\end{array}\right]^{T}$ and $x_{f}(0)=\left[\begin{array}{ll}0 & 0\end{array}\right]^{T}$, the external disturbance is

$$
\omega(t)= \begin{cases}1, & 2 \leq t \leq 3 \\ -1, & 6 \leq t \leq 7 \\ 0, & \text { otherwise }\end{cases}
$$

Then, the simulation results are provided in Figures 1-4. The possible jump mode $\lambda_{t}$ is illustrated in Figure 1. Figure 2 shows the event-triggered release instants and release intervals. Figure 3 plotted the filter state $x_{f}(t)$. The filter error signal $e(t)$ is presented in Figure 4. These simulation results clearly demonstrate that the filter design scheme developed in this paper is effective. 


\section{Conclusion}

The issue of the dissipative filter for SMJSs with time-varying delays has been investigated in this paper. It is should be noted that the event-triggered scheme is employed in order to save the bandwidth resource. The presented scheme provides a sufficient condition to guarantee the resulting filtering error system is stochastically stable and satisfies dissipative. What should be emphasized is that the stability criterion achieved in this paper is less conservative than some existing results. Two numerical examples are given to illustrate the effectiveness and advantages of the proposed filter design scheme. In addition, it is worth mentioning that the scheme developed in this paper can be extended to other dynamic systems, such as neural networks and sampled-data systems with time-varying delays, which will be our future research.

\section{Data Availability}

Data used to support the findings of this work are available from the corresponding author upon request.

\section{Conflicts of Interest}

The authors declare that they have no conflicts of interest.

\section{Acknowledgments}

This work was supported in part by the NSFC (nos. 61573136, 61603133, and 61673169).

\section{References}

[1] O. L. V. Costa and M. V. Araujo, "A generalized multi-period mean-variance portfolio optimization with Markov switching parameters," Automatica, vol. 44, no. 10, pp. 2487-2497, 2008.

[2] A. A. G. Siqueira and M. H. Terra, "A fault-tolerant manipulator robot based on $\mathrm{H}_{2}, \mathrm{H}_{\infty}$, and mixed $\mathrm{H}_{2} / \mathrm{H}_{\infty}$ Markovian controls," IEEE/ASME Transactions on Mechatronics, vol. 14, no. 2, pp. 257-263, 2009.

[3] A. A. G. Siqueira and M. H. Terra, "Nonlinear and Markovian $H_{\infty}$ controls of underactuated manipulators," IEEE Transactions on Control Systems Technology, vol. 12, no. 6, pp. 811-826, 2004.

[4] O. L. V. Costa, M. D. Fragoso, and R. P. Marques, DiscreteTime Markov Jump Linear Systems, Springer, London, UK, 2006.

[5] J. Huang and Y. Shi, "Stochastic stability and robust stabilization of semi-Markov jump linear systems," International Journal of Robust and Nonlinear Control, vol. 23, no. 18, pp. 2028-2043, 2013.

[6] L. Zhang, B. Cai, and Y. Shi, "Stabilization of hidden semiMarkov jump systems: emission probability approach," Automatica, vol. 101, pp. 87-95, 2019.

[7] L. Zhang, Y. Leng, and P. Colaneri, "Stability and stabilization of discrete-time semi-Markov jump linear systems via semiMarkov kernel approach," IEEE Transactions on Automatic Control, vol. 61, no. 2, pp. 503-508, 2016.

[8] H. Shen, Y. Men, Z.-G. Wu, J. Cao, and G. Lu, "Networkbased quantized control for fuzzy singularly perturbed semiMarkov jump systems and its application," IEEE Transactions on Circuits and Systems I: Regular Papers, vol. 66, no. 3, pp. 1130-1140, 2019.

[9] W. Xia, S. Xu, Q. Ma, Z. Qi, and Z. Zhang, "Dissipative controller design for uncertain neutral systems with semiMarkovian jumping parameters," Optimal Control Applications and Methods, vol. 39, no. 2, pp. 888-903, 2018.

[10] H. Shen, F. Li, S. Xu, and V. Sreeram, "Slow state variables feedback stabilization for semi-Markov jump systems with singular perturbations," IEEE Transactions on Automatic Control, vol. 63, no. 8, pp. 2709-2714, 2018.

[11] H. Shen, F. Li, Z.-G. Wu, J. H. Park, and V. Sreeram, "Fuzzymodel-based nonfragile control for nonlinear singularly perturbed systems with semi-Markov jump parameters," IEEE Transactions on Fuzzy Systems, vol. 26, no. 6, pp. 3428-3439, 2018.

[12] T. Yang, L. Zhang, V. Sreeram, A. N. Vargas, T. Hayat, and B. Ahmad, "Time-varying filter design for semi-Markov jump linear systems with intermittent transmission," International Journal of Robust and Nonlinear Control, vol. 27, no. 17, pp. 4035-4049, 2017.

[13] H. Shen, S. Jiao, S. Huo, M. Chen, J. Li, and B. Chen, "On energy-to-peak filtering for semi-Markov jump singular systems with unideal measurements," Signal Processing, vol. 144, pp. 127-133, 2018.

[14] Q. Ma, K. Gu, and N. Choubedar, "Strong stability of a class of difference equations of continuous time and structured singular value problem," Automatica, vol. 87, pp. 32-39, 2018.

[15] W. Xia and S. Xu, "Robust $H_{\infty}$ deconvolution filter for polytopic uncertain systems with distributed delay," Transactions of the Institute of Measurement and Control, vol. 40, no. 11, pp. 3368-3376, 2018.

[16] Y. Li, K. Gu, J. Zhou, and S. Xu, "Estimating stable delay intervals with a discretized Lyapunov-Krasovskii functional formulation,” Automatica, vol. 50, no. 6, pp. 1691-1697, 2014.

[17] B. Zhang, J. Lam, and S. Xu, "Stability analysis of distributed delay neural networks based on relaxed Lyapunov-Krasovskii functionals," IEEE Transactions on Neural Networks and Learning Systems, vol. 26, no. 7, pp. 1480-1492, 2015.

[18] W. Xia, W. X. Zheng, and S. Xu, "Extended dissipativity analysis of digital filters with time delay and Markovian jumping parameters," Signal Processing, vol. 152, pp. 247-254, 2018.

[19] W. Xia, Y. Li, Y. Chu, S. Xu, and Z. Zhang, "Dissipative filter design for uncertain Markovian jump systems with mixed delays and unknown transition rates," Signal Processing, vol. 141, pp. 176-186, 2017.

[20] W. Xia, S. Xu, J. Lu, Y. Chu, Z. Zhang, and Y. Chu, "Reliable filter design for discrete-time neural networks with Markovian jumping parameters and time-varying delay," Journal of the Franklin Institute, 2020, In press.

[21] H. Min, S. Xu, Y. Li, Y. Chu, Y. Wei, and Z. Zhang, "Adaptive finite-time control for stochastic nonlinear systems subject to unknown covariance noise," Journal of the Franklin Institute, vol. 355, no. 5, pp. 2645-2661, 2018.

[22] W. Xia, W. X. Zheng, and S. Xu, "Realizability condition for digital filters with time delay using generalized overflow arithmetic," IEEE Transactions on Circuits and Systems II: Express Briefs, vol. 66, no. 1, pp. 141-145, 2019.

[23] H. Shen, J. Park, Z. Wu, and Z. Zhang, "Finite-time $H_{\infty}$ synchronization for complex networks with semi-Markov jump topology," Communications in Nonlinear Science and Numerical Simulation, vol. 24, no. 1-3, pp. 40-51, 2015.

[24] D. Zhang, J. Cheng, J. Cao, and D. Zhang, "Finite-time synchronization control for semi-Markov jump neural 
networks with mode-dependent stochastic parametric uncertainties," Applied Mathematics and Computation, vol. 344345, pp. 230-242, 2019.

[25] J. Wang, S. Ma, and C. Zhang, "Resilient estimation for T-S fuzzy descriptor systems with semi-Markov jumps and timevarying delay," Information Sciences, vol. 430-431, pp. 104126, 2018.

[26] H. Shen, L. Su, and J. H. Park, "Reliable mixed $H_{\infty}$ /passive control for T-S fuzzy delayed systems based on a semi-Markov jump model approach," Fuzzy Sets and Systems, vol. 314, pp. 79-98, 2017.

[27] H. Shen, Z. Wu, and J. Park, "Reliable mixed passive and $H_{\infty}$ filtering for semi-Markov jump systems with randomly occurring uncertainties and sensor failures," International Journal of Robust and Nonlinear Control, vol. 25, no. 17, pp. 3231-3251, 2015.

[28] F. Li, L. Wu, and P. Shi, "Stochastic stability of semi-Markovian jump systems with mode-dependent delays," International Journal of Robust and Nonlinear Control, vol. 24, no. 18, pp. 3317-3330, 2014.

[29] T. H. Lee, J. Park, S. M. Lee, and O. M. Kwon, "Robust synchronisation of chaotic systems with randomly occurring uncertainties via stochastic sampled-data control," International Journal of Control, vol. 86, no. 1, pp. 107-119, 2013.

[30] X. Zhang and Q. Han, "Event-based $H_{\infty}$ filtering for sampleddata systems," Automatica, vol. 51, pp. 55-69, 2015.

[31] J. Wang, X. Zhang, and Q. Han, "Event-triggered generalized dissipativity filtering for neural networks with time-varying delays," IEEE Transactions on Neural Networks and Learning Systems, vol. 27, no. 1, pp. 77-88, 2016.

[32] Y. Guan, Q. Han, and X. Ge, "On asynchronous event-triggered control of decentralized networked systems," Information Sciences, vol. 425, pp. 127-139, 2018.

[33] W. Xia, W. X. Zheng, and S. Xu, "Event-triggered filter design for Markovian jump delay systems with nonlinear perturbation using quantized measurement," International Journal of Robust and Nonlinear Control, vol. 29, pp. 4644-4664, 2019.

[34] L. Zha, J. Fang, J. Liu, and E. Tian, "Event-based finite-time state estimation for Markovian jump systems with quantizations and randomly occurring nonlinear perturbations," ISA Transactions, vol. 66, pp. 77-85, 2017.

[35] M. S. Aslam and Q. Li, "Quantized dissipative filter design for Markovian switch T-S fuzzy systems with time-varying delays," Soft Computing, vol. 23, no. 21, pp. 11313-11329, 2019.

[36] M. S. Aslam, A. R. Rajput, Q. Li, Y. Liu, and S. A. Niazi, "Adaptive event-triggered mixed $H_{\infty}$ and passivity-based filter design for nonlinear T-S fuzzy markovian switching systems with time-varying delays," IEEE Access, vol. 7, pp. 62577-62591, 2019.

[37] J. Dai and G. Guo, "Event-triggered leader-following consensus for multi-agent systems with semi-Markov switching topologies," Information Sciences, vol. 459, pp. 290-301, 2018.

[38] R. Rakkiyappan, K. Maheswari, G. Velmurugan, and J. Park, "Event-triggered $H_{\infty}$ state estimation for semi-Markov jumping discrete-time neural networks with quantization," Neural Networks, vol. 105, pp. 236-248, 2018.

[39] J. Wang, M. Chen, and H. Shen, "Event-triggered dissipative filtering for networked semi-Markov jump systems and its applications in a mass-spring system model," Nonlinear Dynamics, vol. 87, no. 4, pp. 2741-2753, 2017.

[40] W. Xia, Y. Li, Y. Chu, S. Xu, W. Chen, and Z. Zhang, "Observer-based mixed passive and $H_{\infty}$ control for uncertain Markovian jump systems with time delays using quantized measurements," Nonlinear Analysis-Hybrid Systems, vol. 31, pp. 233-246, 2019.

[41] S. Xu, J. Lam, and X. Mao, "Delay-dependent $H_{\infty}$ control and filtering for uncertain Markovian jump systems with timevarying delays," IEEE Transactions on Circuits and Systems I-Regular Papers, vol. 54, no. 9, pp. 2070-2077, 2007.

[42] L. Chen, X. Huang, and S. Fu, "Observer-based sensor faulttolerant control for semi-Markovian jump systems," Nonlinear Analysis-Hybrid Systems, vol. 22, pp. 161-177, 2016.

[43] W. Xia, Q. Ma, J. Lu, and G. Zhuang, "Reliable filtering with extended dissipativity for uncertain systems with discrete and distributed delays," International Journal of Systems Science, vol. 48, no. 12, pp. 2644-2657, 2017.

[44] A. Seuret, F. Gouaisbaut, and E. Fridman, "Stability of discrete-time systems with time-varying delays via a novel summation inequality," IEEE Transactions on Automatic Control, vol. 60, no. 10, pp. 2740-2745, 2015.

[45] X. M. Zhang, Q. L. Han, and J. Wang, “Admissible delay upper bounds for global asymptotic stability of neural networks with time-varying delays," IEEE Transactions on Neural Networks and Learning Systems, vol. 29, no. 11, pp. 5319-5329, 2018.

[46] K. Liu and A. Seuret, "Comparison of bounding methods for stability analysis of systems with time-varying delays," Journal of the Franklin Institute-Engineering and Applied Mathematics, vol. 354, no. 7, pp. 2979-2993, 2017.

[47] P. G. Park, J. W. Ko, and C. Jeong, "Reciprocally convex approach to stability of systems with time-varying delays," Automatica, vol. 47, no. 1, pp. 235-238, 2011.

[48] D. Yue and Q. Han, "Delay-dependent exponential stability of stochastic systems with time-varying delay, nonlinearity, and Markovian switching," IEEE Transactions on Automatic Control, vol. 50, no. 2, pp. 217-222, 2005.

[49] Z. Fei, H. Gao, and P. Shi, "New results on stabilization of Markovian jump systems with time delay," Automatica, vol. 45, no. 10, pp. 2300-2306, 2009. 